\title{
OPTIMIZED PERFORMANCE WITH PROCESS CHAINS
}

\author{
M. Victoria Hebseeba ${ }^{1}$, Dr. T. Bhaskara Reddy ${ }^{2}$. \\ 1 Department of Computer Science, Rayalaseema University, Andra Pradesh \\ victoria.hebseeba@gmail.com \\ 2 Department of Computer Science, S.K. University, Andra Pradesh \\ bhaskareddy_sku@yahoo.co.in
}

\begin{abstract}
This paper describes the Process chains in SAP Business Warehouse and its main technical features and how the process chains can be used to optimize system efficiency by reducing the usage of system resources or by ensuring that large gaps do not exist between the executions of sequential processes. The approach presented in this paper is best suited to overcome the situations like slowing down the system or processes that are supposed to happen sequentially fail to execute with in the proper timelines when using multiple process chains.
\end{abstract}

\section{Keywords}

Process Type, Variant, Business Warehouse

\section{INTRODUCTION}

A process chain is scheduled sequence of processes linked together and are executed in a specified order. Process chain waits in the background for an event. Some of these processes trigger a separate event that can start other processes in turn. In an operating system there are a multitude of processes in addition to the loading process that occur regularly. Process chain is a mechanism that controls extraction, transformation, and loading (ETL) activities execution at a scheduled time in a defined manner. Process chains are used to automate the complex structures with the help of the controlled processing, visualize the schedule by using network applications and centrally control and monitor the processes.

Process type is the kind of process that is being executed. Each process has a type of activity associated to it. Such as starting an Info package to load data from one object to another, combining two different process chain parts in one depending on the outcome of one of the parts, or starting a program [1]. A process type displays process types, enables to drag and drop process into main panel. A Process has a defined beginning and end and enables openness with regard to the type of process that can be integrated into a process chain. Process chains can be included in other process chains. They are called Meta chain. This gives an option of integrating process chains from the BW system, in which Meta chain is found or from other BW systems.

Transaction code RSPC is to create the process chains and RSPCM to monitor the process chains. We can also create e-mail alert to send email message when the process fails. To create e-mail alert right click on the process and choose maintain message option. Planning view enables to create and modify processes. Checking view checks consistency of a process chain 
selected in plan view. Log view shows log of the execution of a process chain selected in plan view.

Process type determines how the events run in a process chain based on the requirement. Start process describes when the process will start. Is it immediately or scheduled for a job to start the process or through a Meta process. Connector links the processes. When previous process finishes successfully indicates a green arrow. Red indicates a failure. Variant is a set of parameters passed to the processes such as the name of the info package to use for loading, the name of the info objects to perform a change run for[1] [2]. Collector processes allow the designer of a process chain to trigger a subsequent process based on whether certain conditions are met by multiple predecessor processes [5].

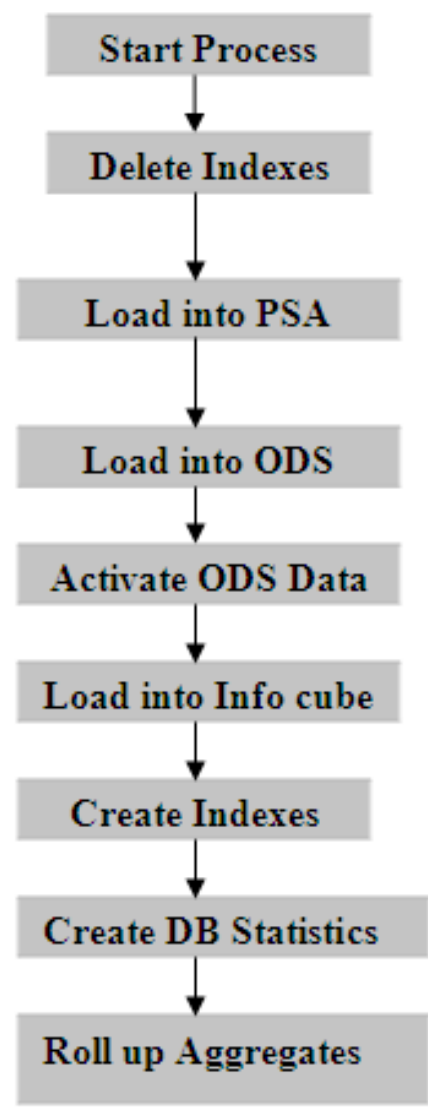

Figure 1: Typical Process chain Architecture

\section{Current Challenge}

A process chain is a mechanism that controls extraction, transformation, and loading (ETL) activities execution at a scheduled time in a defined Sequence.

Since in many support project environments in IT industry multiple process chains are run you come across some issues with the system executing them at appropriate times. The system executes several process chains at once and some times this slows down the system performance. There are situations like process chains that are supposed to happen sequentially fail to execute at proper times. This leaves long time gaps between the process chain executions. 
This slows down the system performance and process chains will not get completed with in the expected time. Business users will be impacted since the latest or most recent data will not be available on time for their reporting purposes.

\section{PROPOSED SOLUTION}

- Convert the update mode from full update mode to delta update mode for the data loads where ever applicable depending on run time so that the run time decreases significantly

- Identify the process chains or data flows which have the full update mode and run time is very high. If the number of records are same on each day, either change the schedule of these loads or move them from daily schedule to weekly or monthly.

- Avoid recursive dependencies that can lead to a dead lock.

- Anomalous situations exist when a process chain stays in an active status while nothing is executing. This status may continue for hours. This happens when you have yellow loading state that continues for hours after the process failed.

- Delay the start of the node for managing process chains executed as part of a master process chain. These Delay nodes ensure the successor process chains start time condition.

- Delay node is applicable for independently scheduled process chains. This delay block supplies the dependency condition by controlling when the predecessor Process chain.

- Process chain containing delay node starts and runs while the first Process chain executes. This is based on analyzing the process chain execution status that the system logs in the RSCLOGCHAIN and RSPCPROCESSLOG tables.

- Table RSPCLOGCHAIN contains log information for the process chain run. Table RSPCPROCESSLOG contains an execution log for each process item.

- The information in the STATE and ACTUAL_STATE columns of the table tells the whole process chain state of each process chain item. Delay node contains ABAP Program. This checks the process chain log tables to check if there are any active predecessor process chains. This checking is repeated till there are no active predecessor logs.

- If Predecessor process chain is successfully finished the next process chain starts. If Predecessor process chain is error finished and if it exceeds the time limit the next process chain does not start.

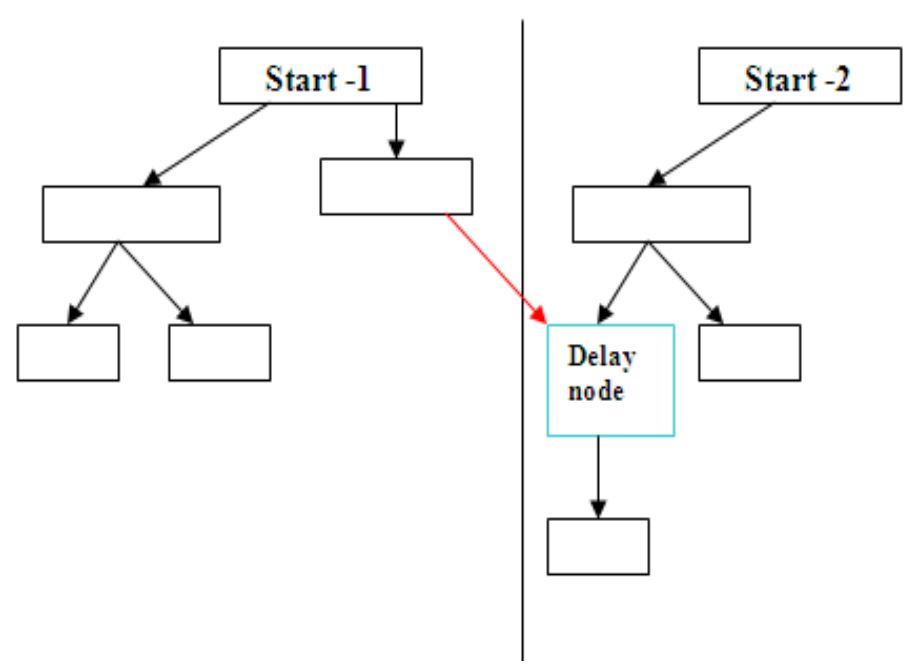

Figure 2: Delay node situation in Process chain 
Advanced Computing: An International Journal ( ACIJ ), Vol.2, No.6, November 2011

\subsection{TEST RESULTS}

- Performed several lab tests with real SAP Net Weaver BI Implementation.

- Developed process chains ZPCH1 and ZPCH2 considering the dependency condition for business critical sales data.

- Scenario-1: Both the process chains were triggered at the same time.

Process chain 1 took 1410 seconds to complete successfully.

Process chain 2 was waiting for Process chain 1 to complete due to the dependency. Process chain 2 took 2842 seconds to complete.

- Scenario-2: Performed changes to the Process chain 2. Implemented delay node approach. Changed the full update mode to delta update for an info package. Process chain 1 tool 1410 seconds to complete successfully.

Process chain 2 has completed in 2017 seconds.

- Developed a reconciliation report to reconcile the data in Info cube and the source data and did not find any duplication or exceptions in the data. Data was consistent in both the scenarios.

Process Chain Execution - Run time in Seconds

$\square$ Process Chain2 $\square$ Process Chain1

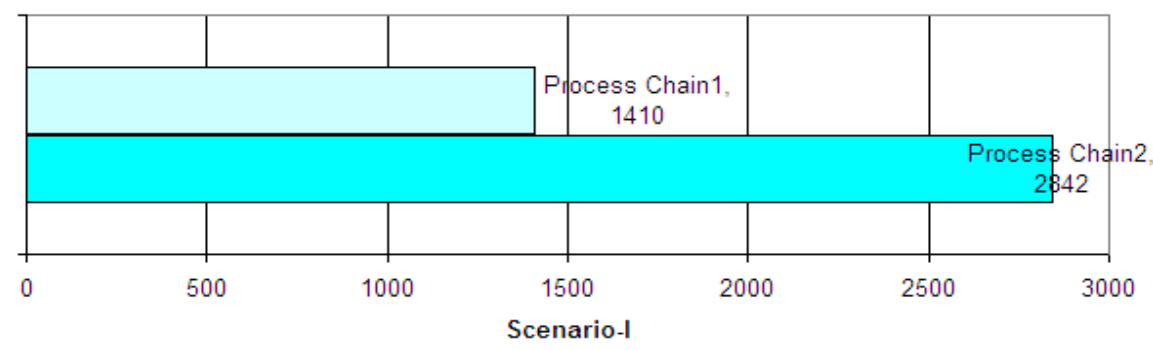

Figure 3: Performance comparison for process chains in Scenario-I

Process Chain Execution - Run time in Seconds

$\square$ Process Chain2 (Modified) $\square$ Process Chain1

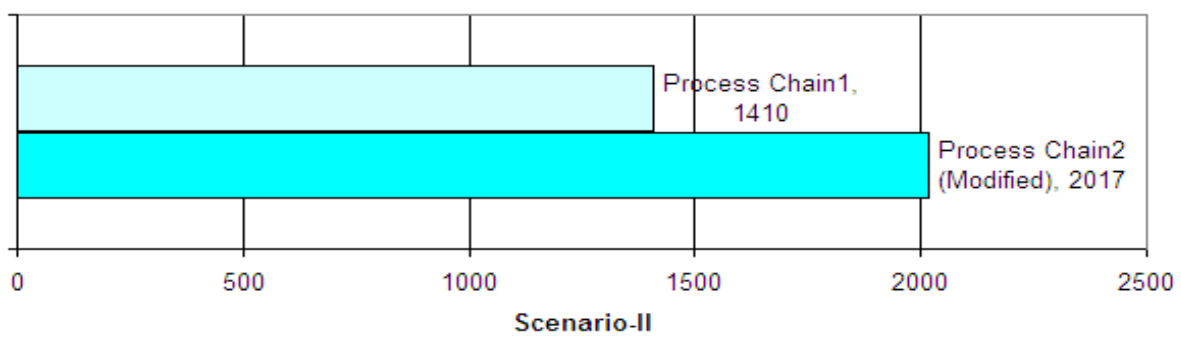

Figure 4: Performance comparison for process chains in Scenario-II

\section{Conclusions}

Process Chains are the new generation of job scheduling and monitoring tools for tasks such as data loads, reporting agent jobs, or index rebuilds.

The proposed approach is best suited for process chain scheduling disturbance caused by anomalous system starts after week end system back up or unplanned system outages since in 
these scenarios some process chains start simultaneously and can cause dead lock situation. How ever this is not a bullet that will resolve every Process chain performance issue. We need to align expectations.

Restructuring of process chains can provide exceptional system performance improvement if applied in the right manner. Various points have been explained and the tests reported in this paper indicate that adapting these approaches offers practical benefits for SAP Net weaver BI support projects and IT Industries.

\subsection{References}

[1] Olaf Klostermann and Milco Osterholm, (2006) "SAP Net weaver BW Administration and Monitoring”, 1st ed., Galileo Press, Germany.

[2] Andrew J. Ross, (2009)" SAP Net weaver BI Accelerator", 1st ed., Galileo Press, Boston (MA), USA.

[3] Christian Merwald, Sabine Morlock, (2009)" Data Ware Housing with SAP BW7, BI in SAP Net Weaver 2004s" 1st ed., Rocky Nook Inc.,

[4] Biao Fu and Henry Fu. (2002) "SAP BW a step by step guide".

[5] Arshad Khan,(2005) "SAP and BW Data Warehousing. How to plan and implement".

[6] Frank K. Wolf and Stefan Yamada, "Data Modeling in SAP Net weaver BW", $1^{\text {st }}$ ed., Galileo Press, Boston,(MA)

[7] Kevin McDonald, Andreas Wilmsmeier, David C. Dixon and W.H Inmon,(2002) "Mastering the SAP Business Information Ware house" Wiley Publishing Inc, USA.

[8] Elizabeth Vitt and Michael Luckevich and StaciaMisner, "Making better Business Intelligence Decisions faster", Microsoft Press, USA

[9] Daniel Knapp, "Practical Data modeling with SAP Net Weaver BW", Galileo Press, Boston(MA), USA

[10] http://airccse.org/journal/ijngn/papers/3111ijngn04.pdf

[11] http://www.ijmo.org/papers/13-P30001.pdf 